\title{
WMSS: A Web-Based Multitiered Surveillance System for Predicting CLABSI
}

\author{
Amin Y. Noaman, ${ }^{1}$ Abdul Hamid M. Ragab (i), ${ }^{2}$ Nabeela Al-Abdullah, ${ }^{3}$ Arwa Jamjoom, \\ Farrukh Nadeem $\left(\mathbb{D},{ }^{2}\right.$ and Anser G. Ali $^{2}$ \\ ${ }^{1}$ Department of Computer Science, Faculty of Computing and Information Technology, \\ King Abdulaziz University, Jeddah, Saudi Arabia \\ ${ }^{2}$ Department of Information Systems, Faculty of Computing and Information Technology, \\ King Abdulaziz University, Jeddah, Saudi Arabia \\ ${ }^{3}$ Clinical Epidemiology \& Infection Control, Faculty of Nursing, King Abdulaziz University, Jeddah, Saudi Arabia
}

Correspondence should be addressed to Abdul Hamid M. Ragab; aragab@kau.edu.sa

Received 13 March 2018; Accepted 6 June 2018; Published 5 July 2018

Academic Editor: Esteban Martinez

Copyright ( $) 2018$ Amin Y. Noaman et al. This is an open access article distributed under the Creative Commons Attribution License, which permits unrestricted use, distribution, and reproduction in any medium, provided the original work is properly cited.

\begin{abstract}
Central-line-associated bloodstream infection (CLABSI) rates are a key quality metric for comparing hospital quality and safety. Manual surveillance systems for CLABSIs are time-consuming and often limited to intensive care units (ICUs). A computerautomated method of CLABSI detection can improve the validity of surveillance. A new web-based, multitiered surveillance system for predicting and reducing CLABSI is proposed. The system has the capability to collect patient-related data from hospital databases and hence predict the patient infection automatically based on knowledge discovery rules and CLABSI decision standard algorithms. In addition, the system has a built-in simulator for generating patients' data records, when needed, offering the capability to train nurses and medical staff for enhancing their qualifications. Applying the proposed system, both CLABSI rates and patient treatment costs can be reduced significantly. The system has many benefits, among which there is the following: it is a web-based system that can collect real patients' data from many IT resources using iPhone, iPad, laptops, Internet, scanners, and hospital databases. These facilities help to collect patients' actual data quickly and safely in electronic format and hence predict CLABSI efficiently. Automation of the patients' data diagnosis process helps in reducing CLABSI detection times. The system is multimediabased; it uses text, colors, and graphics to enhance patient healthcare report generation and charts. It helps healthcare decision makers to review and approve policies and surveillance plans to reduce and prevent CLABSI.
\end{abstract}

\section{Introduction}

Hospital-acquired infections (HAIs), also known as nosocomial infections, are caused by viral, bacterial, and fungal pathogens; the most common types are bloodstream infection (BSI), ventilator-associated pneumonia (VAP), urinary tract infection (UTI), and surgical site infection (SSI), as explained by K. Woeltje et al. [1]. Such infections can be acquired in hospital or in other clinical settings. The infection may be spread by various means to the susceptible patient in the clinical settings. These means may include infected staff, infected patients, contaminated equipment, bed linen, or air droplets. The source of the infection may also come from the outside environment, or from other sources that cannot be determined, or after a surgery that compromises the protective skin barrier. The Centers for Disease Control and Prevention (CDC) estimated roughly 1.7 million hospital-associated infections in the United States from all types of microorganisms. These included bacteria and fungi combined, causing about ninety-nine thousand deaths each year as described by Haidee and Russell [2].

In Europe, where hospital surveys have been conducted, "gram-negative infections are estimated to account for twothirds of the 25,000 deaths each year" [3]. Nosocomial infections can cause severe pneumonia and infections of the urinary tract and bloodstream. Many types of nosocomial 
infections are hard to treat with antibiotics. In addition, antibiotic resistance can complicate treatment.

Central Line Associated Bloodstream Infections (CLABSI) are serious infections typically causing a prolongation of hospital stay and increased cost and risk of mortality. However, "CLABSI can be prevented through proper insertion techniques and management of the central line". These techniques are addressed by the CDC's Healthcare Infection Control Practices Advisory Committee (CDC/HIPAC) [4].

This paper describes a web-based, multitiered surveillance system for predicting and reducing CLABSI. The word "multitiered" means that the system can work using actual patients' real data collected from the web-resources and from hospitals databases. It can also work using patients' data records that can be generated for training by the built-in system simulator; i.e., the system works in two levels of data (actual data and the simulated data). CLABSI is "a primary laboratory confirmed bloodstream infection in a patient with a central line at the time of (or within 48-hours prior to) the onset of symptoms and the infection is not related to an infection from another site" [3]. The blood cultures must be taken from the central line and simultaneously from a peripheral vein, with a shorter time to positivity in the central line sample. Several researchers have tackled the problem of detecting and preventing CLABSI hospital-acquired infections. However, in developed countries it is difficult to get patient data available in electronic formats suitable for use in computing facilities. Even most of the available patients' data in the hospitals are written in paper format and are mostly confidential. To overcome these problems, we have built a simulator to generate CLABSI patients' data records to be able to build and test our proposed system; in addition, it transforms a patient's paper records into an electronic format. This proved very helpful as a system for training healthcare staff members and nurses. The system uses multimedia so that diagnosis reports can be produced in the form of text tables as well as in the form of colored graphics and charts.

The rest of the paper covers the following: Section 2 discusses types of surveillance systems and comparison. Section 3 describes materials and methods. Section 4 explains system results and discussions, and Section 5 is the conclusion.

\section{Surveillance Systems Comparison}

Healthcare-associated infections (HAIs) are a major global public health concern. In developing countries, the lack of surveillance systems leads to an underestimation of the global burden of HAI as explained by Maha Talaat et al. [7]. CLABSIs still occur in intensive care units in developed countries (high income countries) in the US and Europe [4] and in the developing countries (middle/low income countries) in Asia and Africa. Intensive care units (ICUs) in countries with limited resources "have rates of device-associated healthcareassociated infection including CLABSI, VAP, and catheterassociated urinary tract infection (CAUTI) that are 3 to
5 times higher than rates reported from North American, Western European, and Australian ICUs", as explained by Victor D. Rosenthal et al. [8].

Although computer algorithms to detect bloodstream infections can be used to reduce CLABSI, as described by Trick et al. [9], they stated that "automated bloodstream infection surveillance with electronic data is an accurate alternative to surveillance with manually collected data". Also, a comparison of automated strategies for surveillance of nosocomial bacteremia was investigated by Cristina Bellini et al. [10]. They concluded that automated strategies are convenient alternatives to manual surveillance of nosocomial BSI. An automated CLABSI surveillance approach has also several advantages, including reducing the time spent by infection control specialists on routine surveillance and reallocating it to prevention efforts and potentially extending surveillance outside of the intensive care unit (ICU) [11].

Automated algorithmic approaches for translating manual infection control surveillance methods were explained by Bala Hota et al. [12]. The goal was to automat infection detection that would achieve the CDC aim and to improve the financial support for hospitals to deploy electronic health records.

A semiautomated electronic surveillance system for ventilator-associated pneumonia (VAP) and CLABSI in a Dutch intensive care unit was described by Anna Maria Kaiser [13]. They concluded that surveillance of VAP and CLABSI with a trigger-based electronic surveillance system (ESS) is feasible and effective because of its time-saving benefits. An automated surveillance system was described by Keith F. Woeltje [14]; it uses existing databases with patient-level variables and microbiology data. It can perform acceptably good surveillance in areas where resources do not allow for traditional manual surveillance.

Due to the unavailability of patient data and confidentiality issues, specifically in developing countries simulated patient records can be used. Simulation-based training associated with reduced CLABSI rates was used by Gilman B. Allen [15]. In their medical and surgical intensive care units, they obtained the benefits of simulation training, electronic medical records, and standardized kits to reduce their CLABSI rates. The several benefits of CLABSI simulation training were explained by Karla M. Abela [16], among which there is the following: learning environment is safe. Teaching methodology is flexible. Patient safety is not compromised. Feedbacked data can be structured immediately. Student focused learning may be individualized. Simulation-based training can significantly improve nurses' education quality and hence leads to reduced CLABSI rates as explained by Khouli $\mathrm{H}$ et al. [17] and by Barsuk JH. et al. [18]. Also, simulation training can produce significant medical care cost savings, as explained by Elaine R. Cohen [19].

\section{Materials and Methods}

This section describes the architecture of the proposed system, including materials used and methods. 
TABLE 1: Primary bloodstream infection (BSI) form and instructions for completion (CDC 57.108) [3].

\begin{tabular}{|c|c|}
\hline Data Field & Instructions for Data Collection \\
\hline Facility ID & It will be auto entered by the computer. \\
\hline Event \# & It will be auto entered by the computer. \\
\hline Patient ID & $\begin{array}{l}\text { Required. Enter the alphanumeric patient ID number. } \\
\text { It consists of any combination of numbers and/or letters. }\end{array}$ \\
\hline Social Security \# & Optional. Enter the 9-digit numeric patient Social Security Number. \\
\hline Secondary ID & $\begin{array}{l}\text { Optional. Enter the alphanumeric ID number assigned } \\
\text { by the facility. }\end{array}$ \\
\hline Medicare \# & $\begin{array}{l}\text { Conditionally required. Enter the patient's Medicare number for all events reported as part of a CMS Quality } \\
\text { Reporting Program. }\end{array}$ \\
\hline Patient name & Optional. Enter the last, first, and middle name of the patient. \\
\hline Gender & $\begin{array}{l}\text { Required. Check Female, Male, or Other to indicate the } \\
\text { gender of the patient. }\end{array}$ \\
\hline Date of Birth & Required. Use this format: MM/DD/YYYY. \\
\hline Ethnicity & Optional. \\
\hline Race & Optional. \\
\hline Event type & Required. BSI. \\
\hline Date of event & $\begin{array}{l}\text { Required to meet the BSI criterion occurred for the first time, during the Infection Window Period, using this } \\
\text { format: MM/DD/YYYY. }\end{array}$ \\
\hline
\end{tabular}

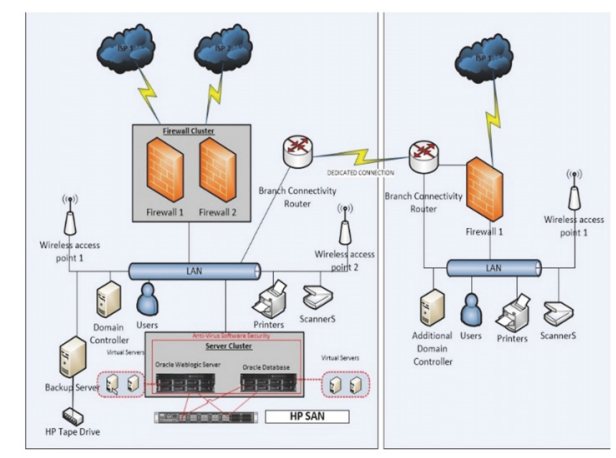

FIGURE 1: The system hardware web components developed.

3.1. System Hardware Components. Figure 1 shows the system hardware web components developed, based on oracle architecture. To ensure system data protection activity and to ensure security and safety, the following steps has been implemented:

(1) Users are connected to the Hospital Servers through firewalls.

(2) Users are connected to fill data through a secure Java Web start application maintained by Oracle WebLogic Server.

(3) Data and application stores in Server Cluster are protected using Antivirus Software Security.

(4) Also, the data is secured by Default Oracle Database Security.
(5) The Clinical Infections Department at King Abdulaziz University Hospital was connected to the system during the experimental stage; it covers 750 beds.

(6) Several local hospitals are connected to the system during the experimental stage.

3.2. System Software Components. The proposed system software is constructed of six components (modules), as shown in Figure 2. These include the Patients' Data Module (PDM), the CLABSI algorithm Module (CAM), the Symptoms and Criteria Module (SCM), the Risk Indicators Module (RIM), the Contamination Module (CM), and the Simulator module (SM). The functions of these components are described in the following sections, respectively.

3.2.1. Patients' Data Module (PDM). This software component is responsible for collecting patients' data from several resources from hospitals locally or remotely from the portal web site. This can be done using many IT resources including iPhones, iPad, laptops, the Internet, scanners, and hospital databases. Table 1 shows a part of the primary bloodstream infections entry form and instructions for completion [20]. To detect if a patient has a CLABSI infection or not, additional patient data including symptoms criteria is inputted to the surveillance system proposed, as explained in the next sections.

3.2.2. CLABSI Algorithm Module (CAM). This system software component is responsible for applying the standard CLABSI algorithm as shown in Figure 2. It is used for diagnosis patients' data collected. A positive blood culture 


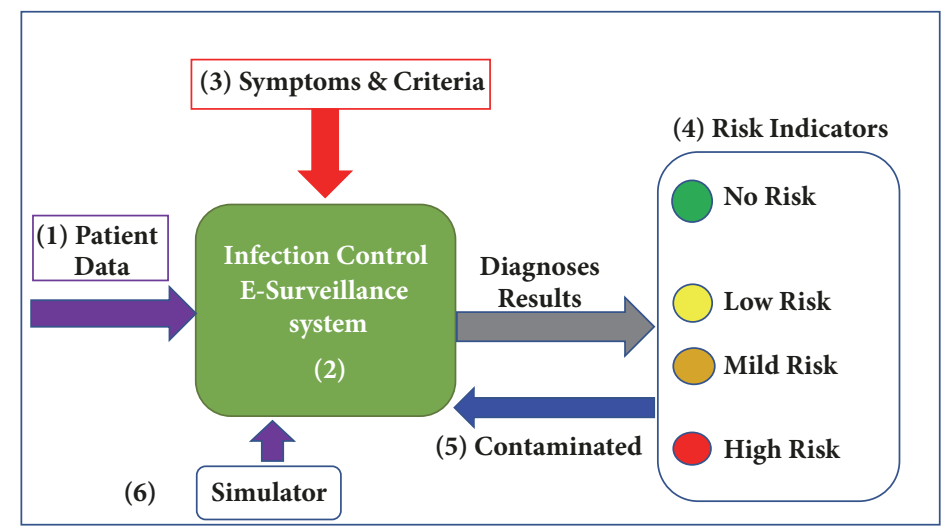

FIgURE 2: The proposed WMSS-CLABSI system software components.

with signs and symptoms of infection are evidence of a bloodstream infection. A patient may develop a CLABSI case, when

(1) s/he suffers from fevers, chills, or the skin around the catheter may become sore and red.

(2) When a bloodstream infection occurs and there is no other place where that infection could be coming from.

(3) The infection is associated with the central line.

(4) It is still possible that the infection could be coming from another place but that source is hidden [5].

The CLABSI algorithm deals with these symptoms to know and detect the type of infection, as explained in Section 3.2.3.

3.2.3. Symptoms and Criteria Module (SCM). This system software component is responsible for finding out CLABSI symptoms and evaluating their criteria, for diagnosis of patients' data. We built the list of CLABSI symptoms and criteria based on hospital-acquired infection definition described in $[5,20]$. In our experimental stage, we have applied twenty-four CLABSI predictors that are shown in Table 2 . They can be used effectively for predicting patients' infections.

3.2.4. Risk Indicators Module (RIM). This system software component is utilized to estimate patient illness due to predicted infection, as shown in Table 3.

The risk indicator software module computes total symptoms weights and indicates CLABSI prediction results, in the form of colors, as shown in Table 3. Table 4 shows CLABSI predictors and symptoms in detail and the VALUE-LABLE risk weights (high, mild, low, and no risk) used for infection prediction. Four levels of infection indicators (A, B, C, and D) are proposed. Red color indicates CLABSI high risk (A), when sum of high symptoms criteria weights was more than or equal five. Orange color indicates CLABSI mild risk (B) when sum of mild symptoms criteria weights was more than or equal five. Yellow color indicates CLABSI low risk (C) when sum of low symptoms criteria weights was more than or equal five. Green color indicates no risk (D) when sum of normal symptoms criteria weights was more than or equal five. At least the sum of a total of five symptoms criteria should be achieved to justify an infected prediction for CLABSI patient cases.

Using of color indicators are an appropriate classification of the patients' risk of developing CLABSI, because it is based on the electronic hospital-acquired infection surveillance Phoenix system used at KAUH, which uses colors. And we established it based on other classification, such as ASA (American Society of Anesthesiologists) physical status classification system [21]. Also, the color indicators are suitable for providing a quick vision test, for indicator that can be figured out by staff members and nurses, specifically suitable in case of training. In addition, the system can also produce detailed results of patients' diagnosis data in the form of tables and charts, as explained in next sections.

3.2.5. Contamination Module (CM). This system software component is responsible for producing feedback information after a diagnosis of a CLABSI patient case. It tells the nurses and medical staff whether the patient is now infected or not. If a patient is infected, patient treatment by staff and nurses should be carried out quickly to cure the patient safely. Then, the system continues to remeasure the patient case, until s/he is recovered from the infection.

3.2.6. Simulator Module (SM). This system software component was built to simulate patient data records automatically, when required for training. It is used for testing the system's CLABSI prediction and patient data validation. In addition, it is used for nurses' education and medical staff training. To proof that the simulator generates patients' data records and gives almost identical results as the actual patients' data records, the following steps are implemented:

(1) The simulator generates patient prediction records randomly. These records are generated by Oracle database procedure. 
TABLE 2: Summary of CLABSI predictors.

List of predictions:

(1) Multiple insertion.

(2) Site of insertion (femoral, subclavian, jugular).

(3) Rewire (Yes or No).

(4) Status of Procedure (Elective or Emergency).

(5) Co-morbidities (drop down selections):

(a) DM (Diabetes Mellitus),

(b) HTN (Hypertension),

(c) BA (Bronchial Asthma),

(d) Malignancy,

(e) Heart Disease etc.

(6) Compliant to Central Line Insertion \& Maintenance bundle (Yes or No).

(7) Length of stay (>14 days).

(8) Parenteral nutrition (Yes or No).

(9) Antimicrobial usage (Yes or No).

(10) Renal failure (Yes or No).

(11) Surgical procedure (Yes or No).

(12) Age.

(13) Sex (Male or Female).

(14) Duration of device use.

(15) Transfer from other hospital (Yes or No).

(16) Transfer from other unit within the hospital (Yes or No).

(17) Co-existing infection (Yes or No).

(18) Temperature: (on admission \& 48 hrs. after insertion).

(19) APACHE Score: 0-14(MILD); 15-29(MODERATE); $\geq 30$ (SEVERE).

(20) Ventilated (Yes or No).

In addition, for neonates \& children.

(21) Birth weight ( $\leq 750 \mathrm{gm} ; 750-1000 \mathrm{gm} ; 1001-1500 \mathrm{gm} ; 1501-2500 \mathrm{gm}$; >2500gm).

(22) Nasal CPAP (Yes or No).

(23) Blood Transfusion (Yes or No).

(24) Trauma (Yes or No).

TABLE 3: CLABSI indicators due to total symptom weightings.

\begin{tabular}{lccc}
\hline Patient case Category & Symptoms weight & Type of Risk & Color Indicator \\
\hline A & If sum of High symptoms & high risk & Red \\
& Criteria weights $>=5$ & mild risk & Orange \\
B & If sum of Mild symptoms & low risk & Yellow \\
& Criteria weights $>=5$ & & no risk \\
C & If sum of low symptoms & Green \\
& Criteria weights $>=5$ & If sum of symptoms & \\
\hline
\end{tabular}

(2) The prediction values are selected from a predefined set of values. These procedure uses the default Oracle random procedure.

(3) The interdependencies of predictors have been implemented (like birth weight predictor links with newborn predictor, parenteral nutrition links with gender or multiple insertion with status of procedure).
(4) By default, the procedure generates 10 patients' records. To proof that simulation results are equivalent and almost identical into real results, we apply the same algorithms and formulas used in the case of actual data.

(5) The simulator algorithm checks if the predictor's values are valid as the actual values, then results will be accepted. 


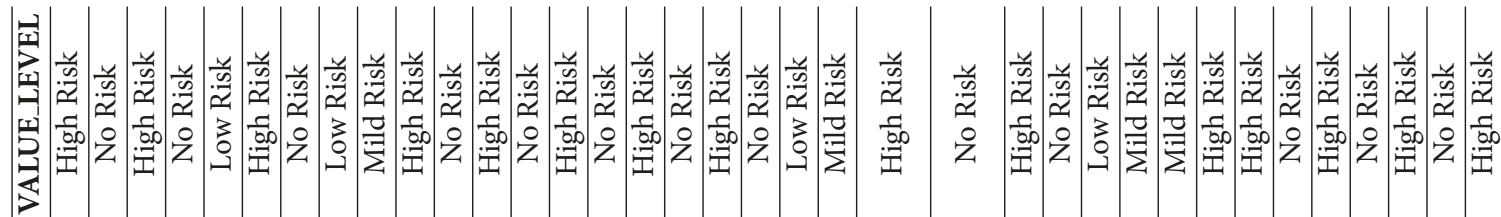

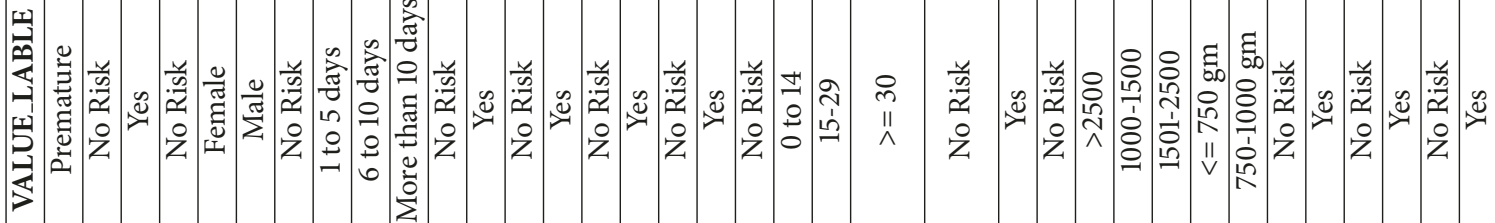

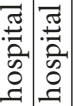
吾焉焉焉

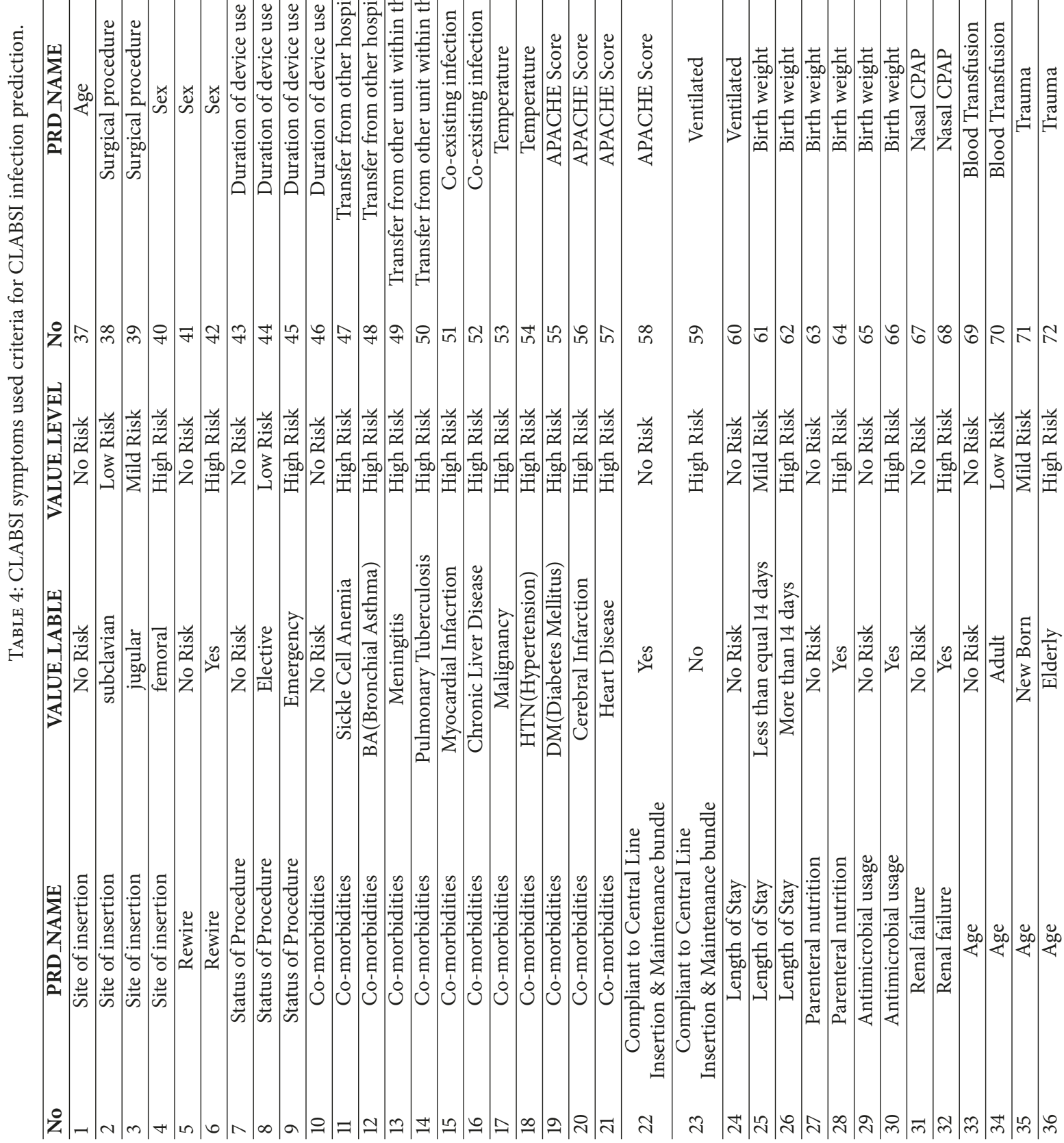




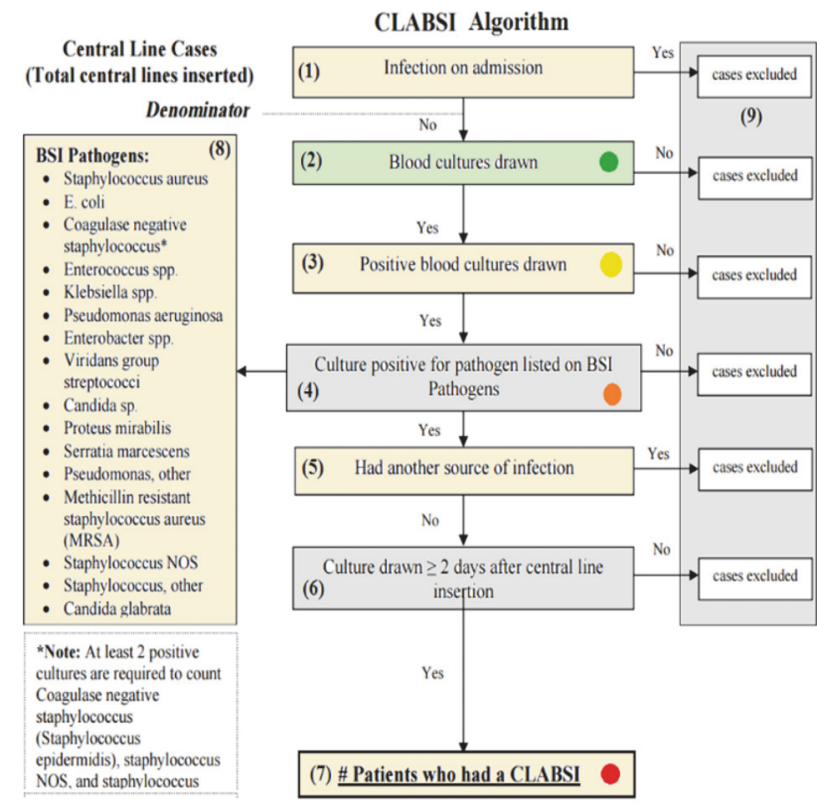

FIgURE 3: The standard CLABSI algorithm adapted from $[5,6]$.
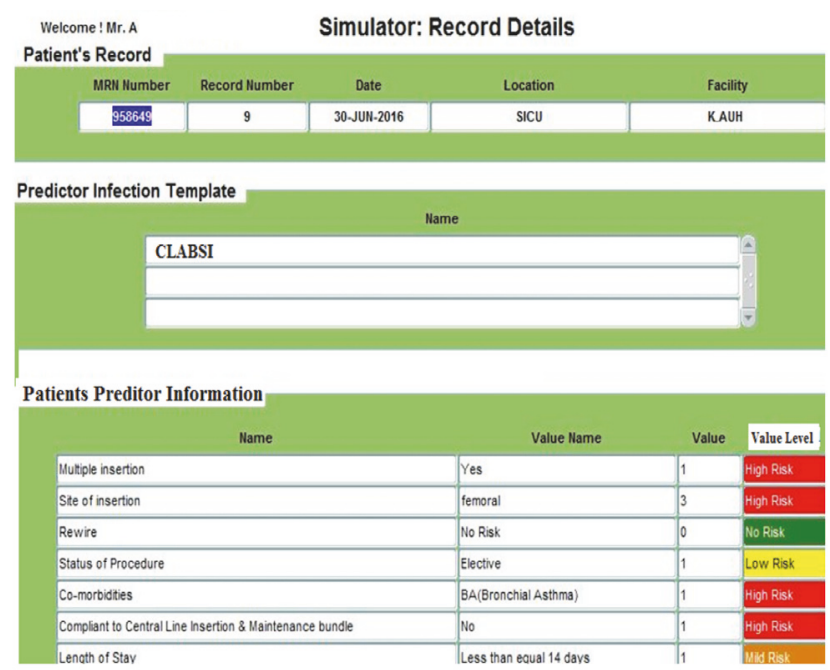

FIgURE 4: A sample of a simulator generated patient record.

Figure 3, for example, shows a sample of a simulator generated patient records. When comparing this simulated patient data records with the actual real data records, as shown in Figure 5, it indicates that the results obtained are identical.

\section{Results and Discussions}

4.1. Patient Information Input Screen. The results explained here are based on real patients' data from King Abdulaziz University Hospital (KAUH), containing 750 beds, dept. of clinical infection control. Figure 4 shows the patient information input screen. Figure 5 shows the event details input screen. The built-in simulator is an additional facility

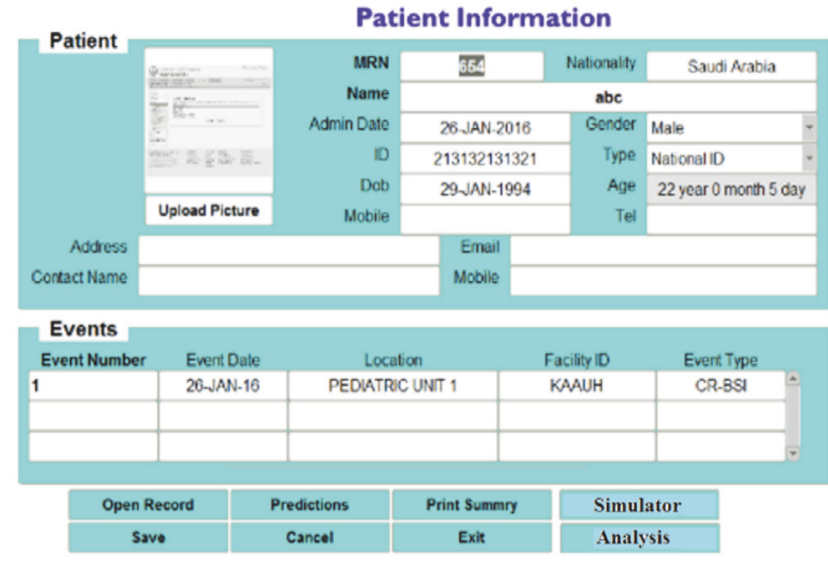

FIgURe 5: Patient information data entry form.

for generating artificial patient data records that can be used when needed by staff and nurses to input simulated patient data records for training and education. The proposed WMSS-CLABSI system produces several types of charts, graphs, tables, and reports of patients' information, including infection detection and diagnosis. Figure 6 shows patient event details of the data entry form. Figure 7 shows a sample of patient record details and patient predictors information.

4.2. Reports Generated and Risk Prediction. Figure 8 shows a sample of the generated prediction summary report. It indicates patient's number of records, event dates, and symptoms with diagnosis indicators. Based on these patients' information, the percentage of infections risk can be computed as indicated in Table 5. It shows the theoretically percentage of types of CLABSI risk for each group (defined in Table 4) including high, mild, low, and normal risks. Each cell value in Table 5 is computed by dividing predicted numbers of CLABSI risk types by total symptoms (24) multiplied in 100 .

4.3. Device Utilization and Standard Infection Ratios. Figures 9(a) and 9(b) show a bar graph chart indicating the relationship between Central Line Days (CLDs), Patient Days (PDs), and Device Utilization Ratio (DUR) as a function of time (in months). For example, it shows that, during July, the CLDs $=85$ and $P D s=153$, then DUR $=85 / 153=0.38$.

Figures 10(a) and 10(b) show some results using bar chart graphs for computing the standard infection ratio (SIR) as a function of time. SIR is computed by dividing CLABSI observed cases over CLABSI predicted cases. It is one of the important measures for judging the quality of a hospital's CLABSI detection and prevention. The standard value of SIR is one. When SIR has a value below one it indicates better hospital safety and vice versa. The higher ratio of SIR reflects a need for stronger CLABSI prevention efforts, while the lower SIR ratio reflects a robust CLABSI prevention strategies [22].

Figure 10(b) shows that there is a regularly smooth reduction in SIR ratio, as function of time (in months). This is a good indication factor. The average SIR ratio within the $1^{\text {st }}$ six months (from Jan. up to Jun.) is 0.86 , 
TABLE 5: Percentage of the several categories of CLABSI risk.

\begin{tabular}{|c|c|c|c|c|c|}
\hline \multicolumn{6}{|c|}{ Percentage of Risk of CLABSI for Each Group } \\
\hline MRN & High & Mild & Low & Normal & Final \\
\hline 900088 & $12 / 24=50 \%$ & $2 / 24=8.44 \%$ & $1 / 24=4.17 \%$ & $9 / 24=37.5 \%$ & High \\
\hline 900096 & $4 / 24=16.67 \%$ & $1 / 24=4.17 \%$ & $5 / 24=20.83 \%$ & $14 / 24=58.33 \%$ & Low \\
\hline 900100 & $4 / 24=16.67 \%$ & $5 / 24=20.83 \%$ & $2 / 24=8.44 \%$ & $13 / 24=54.17 \%$ & Mild \\
\hline 900124 & $4 / 24=16.67 \%$ & $4 / 24=16.67 \%$ & $3 / 24=12.5 \%$ & $13 / 24=54.17 \%$ & Normal \\
\hline 900203 & $9 / 24=37.5 \%$ & $1 / 24=4.17 \%$ & $3 / 24=12.5 \%$ & $11 / 24=45.83 \%$ & High \\
\hline 900593 & $16 / 24=66.67 \%$ & $1 / 24=4.17 \%$ & $2 / 24=8.44 \%$ & $5 / 24=20.83 \%$ & High \\
\hline 900653 & $12 / 24=50 \%$ & $2 / 24=8.44 \%$ & $1 / 24=4.17 \%$ & $9 / 24=37.5 \%$ & High \\
\hline 900753 & $11 / 24=45.83 \%$ & $3 / 24=12.5 \%$ & $3 / 24=12.5 \%$ & $7 / 24=29.17 \%$ & High \\
\hline
\end{tabular}

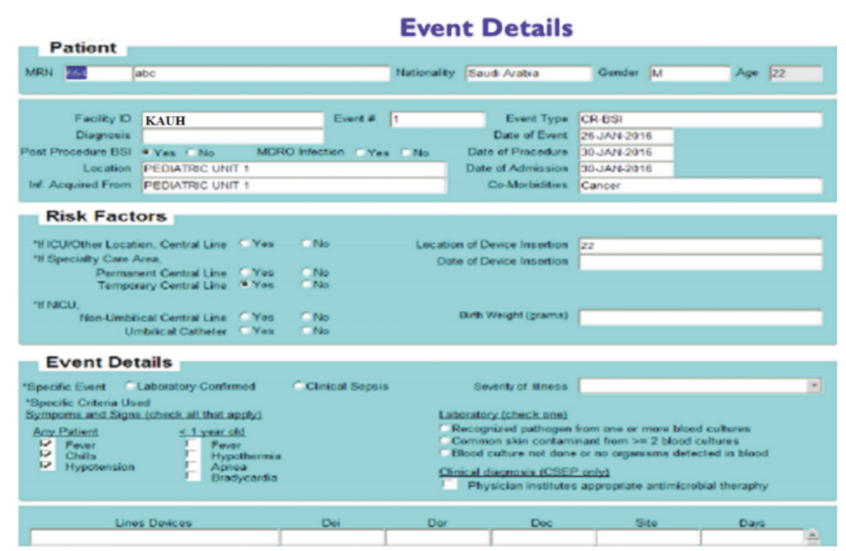

Figure 6: Patient event details data entry form.
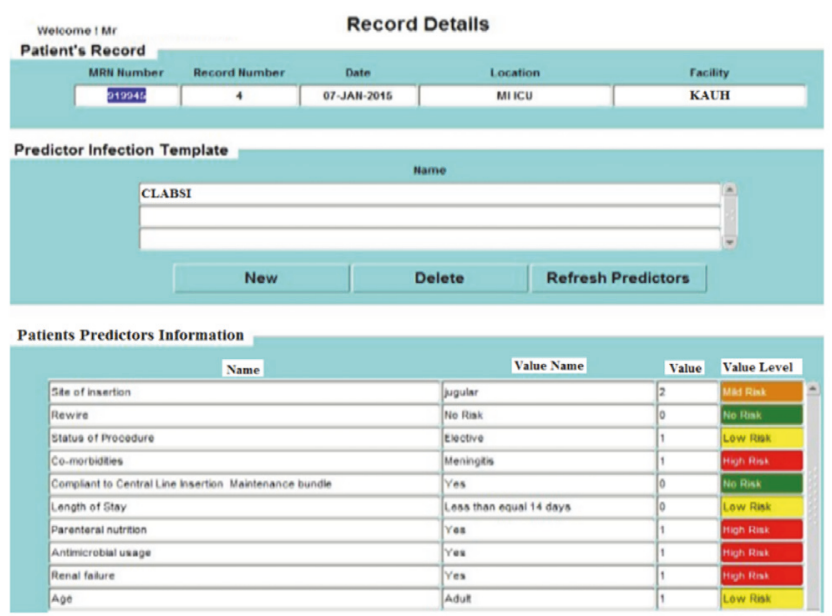

Figure 7: Sample of patient record details and predictors information.

based on CLABSI traditional manual method used. And during the next six months (from Jul. up to Dec.), the average SIR ratio within these six months is 0.73 , where the automated proposed method was used. These results give a reduction in the SIR ratio $=86-73=13 \%$. Hence, the system improvement value $=87 \%$. This proves that the proposed automated system gives improvement in CLABSI prediction performance better than the traditional manual method. Hence, based on these results, applying the proposed method achieves better regular reduction in SIR, as function of time in months, and better than that achieved by the manual method.

\section{Conclusions}

This paper presented a new web-based, multitiered surveillance system for predicting central line associated bloodstream infections (CLABSI). The system collects patients' 


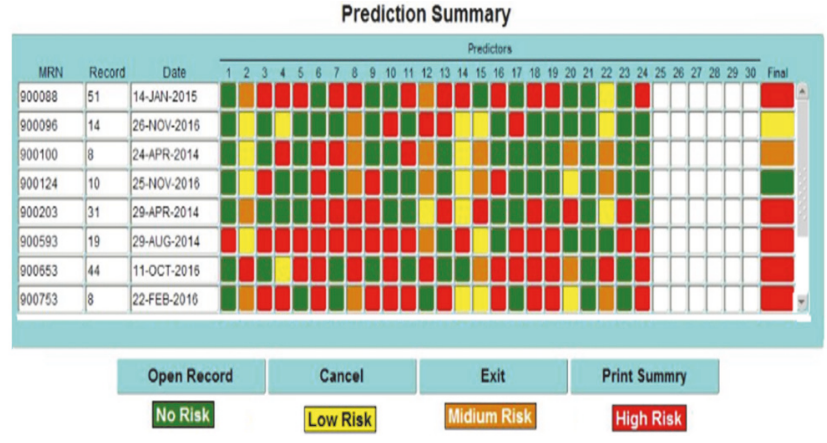

FIGURE 8: A sample of the generated prediction summary report.

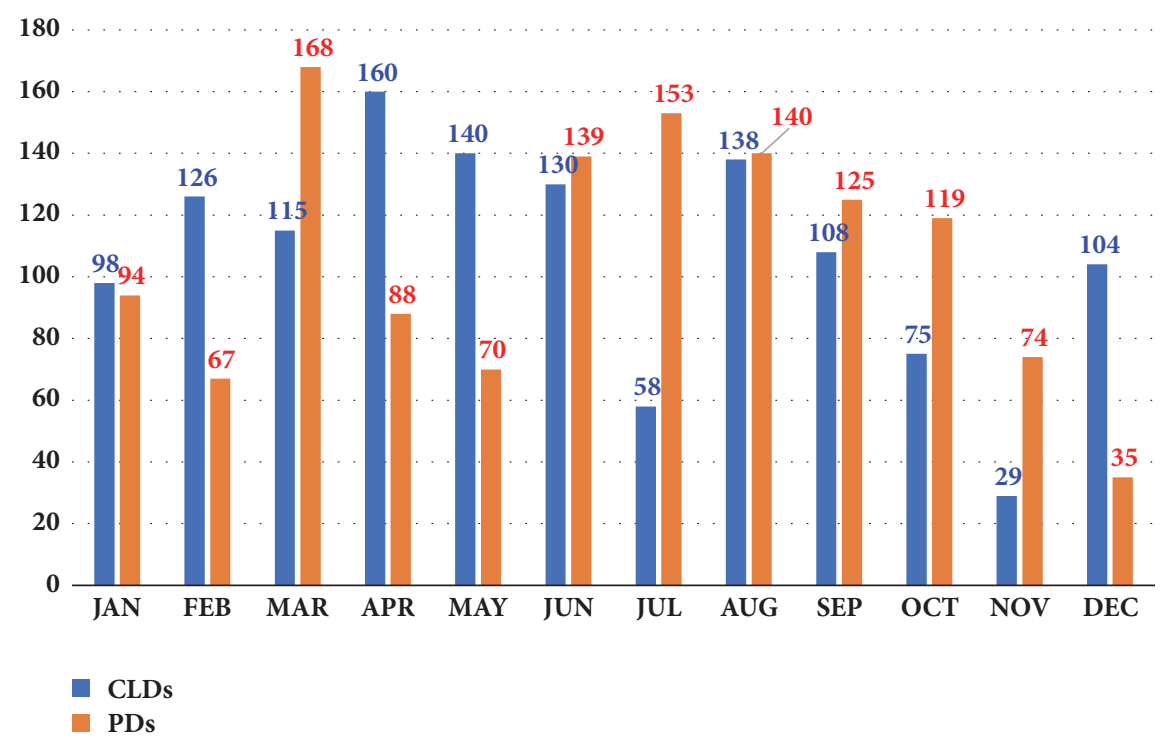

(a) Relation between Central Line Days (CLDs) and Patient Days (PDs) as a function of time

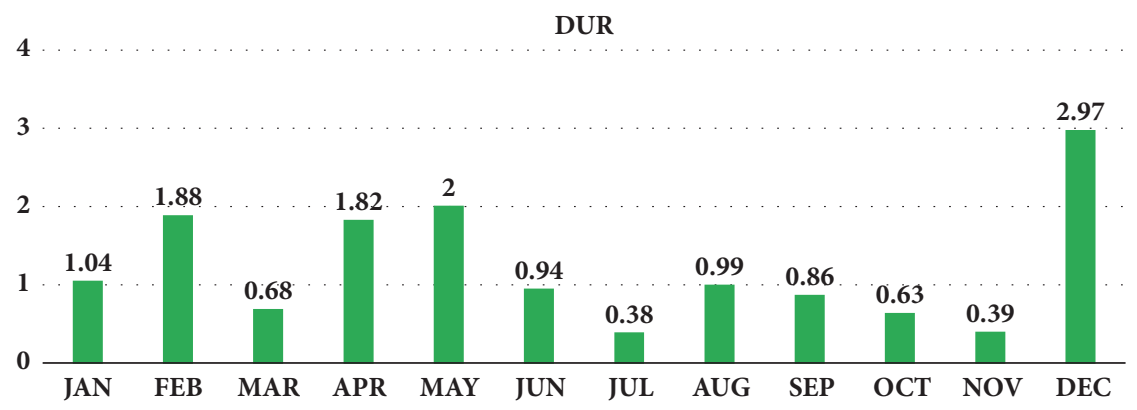

(b) Device Utilization Ratio (DUR) as a function of time

FiguRE 9

data from several resources including laptops, Internet, scanners, and hospitals databases. This process helps hospitals to gather patients' data efficiently and accurately. Patients' records are automatically processed for CLABSI diagnoses and prediction based on knowledge discovery CLABSI algorithms. This helps to reduce the process of infection prediction time and hence reduce CLABSIs rates. The outputs of predicted infected cases are produced using visualized multimedia forms including text reports, colored risk indicators, and graphic charts. This helps medical decision makers to review and approve policies for surveillance plans. The system has also a built-in simulator to simulate specific patient data records, when needed, for nurses' training and education. This helps in reducing and preventing many CLABSIs cases and hence keeps a hospital safe while reducing treatment costs. We are currently expanding the WMSS-CLABSI system to include ventilator-associated pneumonia (VAP), urinary tract infection (UTI), and surgical site infection (SSI), 


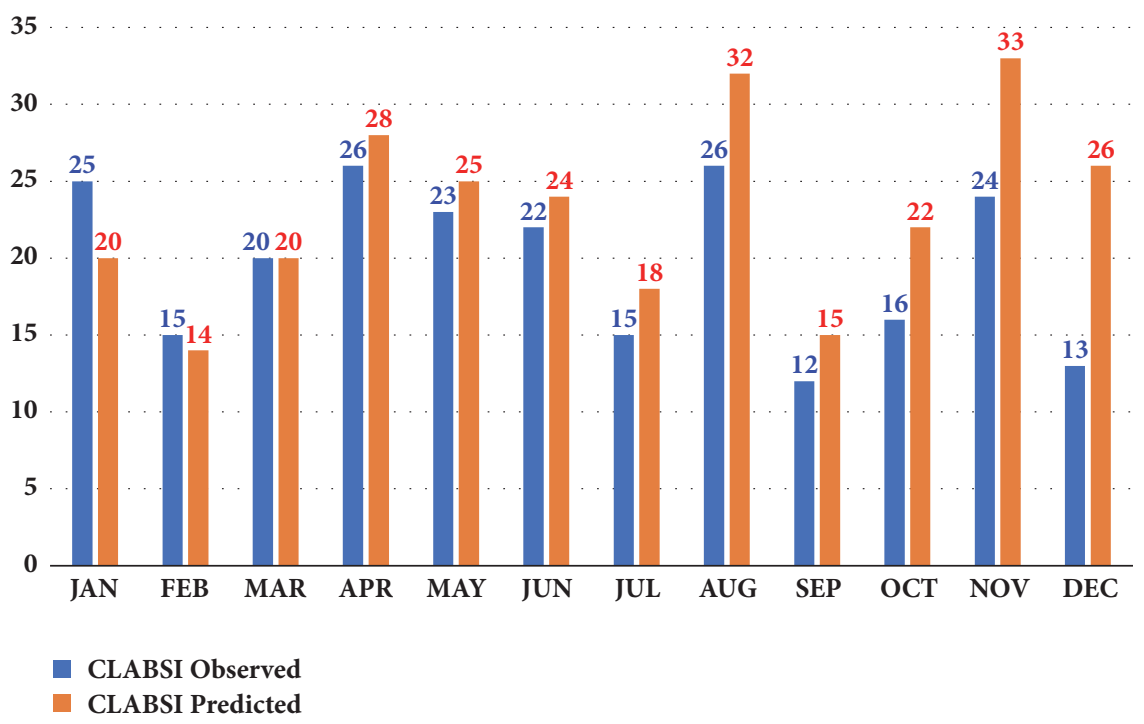

(a) CLABSI observed and CLABSI predicted as a function of time

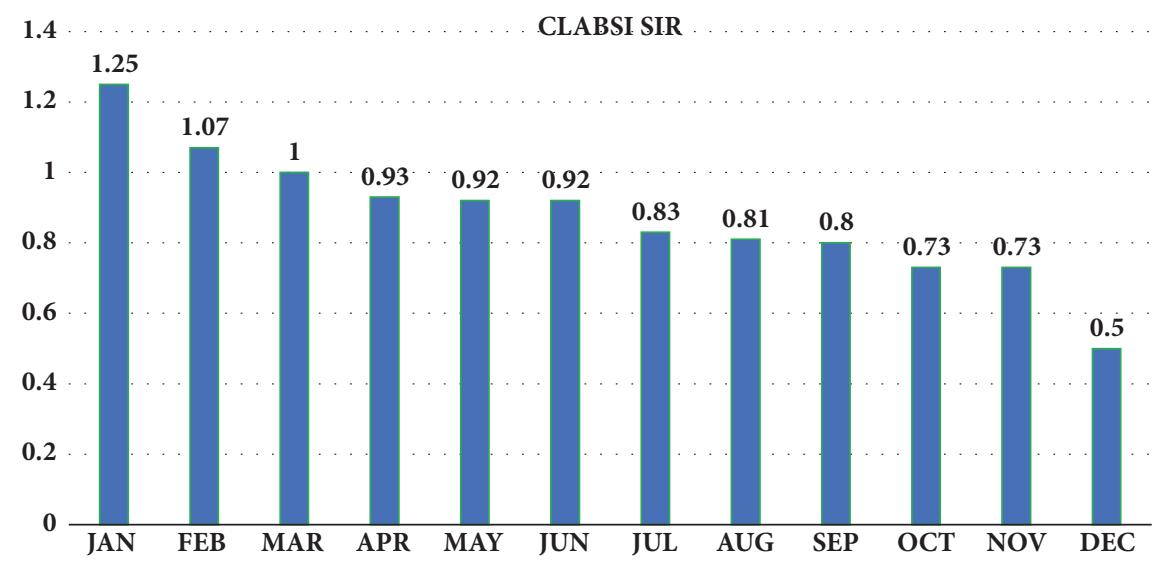

(b) The standard infection ratio (SIR) as a function of time

Figure 10

at King Abdulaziz University Hospital, in Jeddah, Saudi Arabia.

\section{Data Availability}

The data used to support the findings of this study are available from the corresponding author upon request.

\section{Conflicts of Interest}

The authors declare that they have no conflicts of interest.

\section{Acknowledgments}

This project was funded by the National Plan for Science, Technology and Innovation (MAARIFAH), King Abdulaziz City for Science and Technology, Saudi Arabia, Award no. 12-INF2752-03. The authors also thankfully acknowledge
Science and Technology Unit, King Abdulaziz University, for technical support.

\section{References}

[1] K. F. Woeltje, M. Y. Lin, M. Klompas, M. O. Wright, G. Zuccotti, and W. E. Trick, "Data requirements for electronic surveillance of healthcare-associated infections," Infection Control and Hospital Epidemiology, vol. 35, no. 9, pp. 1083-1091, 2014.

[2] T. H. Custodio and W. R. Steele, "Hospital Acquired Infections," 2016 [cited 2017 March 15]; 1-7, http://emedicine .medscape.com/article/967022-overview.

[3] The Centers for Disease Control and Prevention (CDC), 19 April 2017 [cited 2017 April 20]; 1-10, https://en.wikipedia .org/wiki/Centers_for_Disease_Control_and_Prevention.

[4] National Healthcare Safety Network (NHSN) Overview CDC, Jan 2017 [cited 2017 April 20]; 1-4, https://www.cdc .gov/nhsn/pdfs/pscmanual/1psc_overviewcurrent.pdf. 
[5] Central Line-associated Bloodstream Infection (CLABSI), March 2016 [cited 2017 April 20], https://www.cdc.gov/hai/ bsi/bsi.html.

[6] Medicare Patient Safety Monitoring System Measure Algorithms, October 2015 [cited 2017 April 20], https://www.rairarubiabooks.com/related-pdf-medicare-patient-safety-monitoring-system-mpsms.html.

[7] M. Talaat, M. El-Shokry, J. El-Kholy et al., "National surveillance of health care-associated infections in Egypt: developing a sustainable program in a resource-limited country," American Journal of Infection Control, vol. 44, no. 11, pp. 1296-1301, 2016.

[8] V. D. Rosenthal, D. G. Maki, and N. Graves, "The International Nosocomial Infection Control Consortium (INICC): goals and objectives, description of surveillance methods, and operational activities," American Journal of Infection Control, vol. 36, no. 9, pp. el-e12, 2008.

[9] W. E. Trick, B. M. Zagorski, J. I. Tokars et al., "Computer algorithms to detect bloodstream infections," Emerging Infectious Diseases, vol. 10, no. 9, pp. 1612-1620, 2004.

[10] C. Bellini, C. Petignat, P. Francioli et al., "Comparison of automated strategies for surveillance of nosocomial bacteremia," Infection Control and Hospital Epidemiology, vol. 28, no. 9, pp. 1030-1035, 2007.

[11] K. F. Woeltje, A. M. Butler, A. J. Goris et al., "Automated surveillance for central line-associated bloodstream infection in intensive care units," Infection Control and Hospital Epidemiology, vol. 29, no. 9, pp. 842-846, 2008.

[12] B. Hota, M. Lin, J. A. Doherty et al., "Formulation of a model for automating infection surveillance: algorithmic detection of central-line associated bloodstream infection," Journal of the American Medical Informatics Association, vol. 17, no. 1, pp. 4248, 2010.

[13] A. M. Kaiser, E. de Jong, S. F. Evelein-Brugman, J. M. Peppink, C. M. Vandenbroucke-Grauls, and A. R. Girbes, "Development of trigger-based semi-automated surveillance of ventilatorassociated pneumonia and central line-associated bloodstream infections in a Dutch intensive care," Annals of Intensive Care, vol. 4, no. 40, pp. 1-7, 2014.

[14] K. F. Woeltje, K. M. McMullen, A. M. Butler, A. J. Goris, and J. A. Doherty, "Electronic surveillance for healthcare-associated central line-associated bloodstream infections outside the intensive care unit," Infection Control and Hospital Epidemiology, vol. 32, no. 11, pp. 1086-1090, 2011.

[15] G. B. Allen, V. Miller, C. Nicholas et al., "A multitiered strategy of simulation training, kit consolidation, and electronic documentation is associated with a reduction in central line-associated bloodstream infections," American Journal of Infection Control, vol. 42, no. 6, pp. 643-648, 2014.

[16] K. M. Abela, "Using Simulation to Improve CLABSI Prevention in Pediatrics," Nov 2012 [cited 2017 April 20], https://www .eiseverywhere.com/file_uploads/f287el8d6caf520d55dc2aa9510a35ba_SC206Sa5ABELA.pdf.

[17] H. Khouli, K. Jahnes, J. Shapiro et al., "Performance of medical residents in sterile techniques during central vein catheterization randomized trial of efficacy of simulation-based training," CHEST, vol. 139, no. 1, pp. 80-87, 2011.

[18] J. H. Barsuk, E. R. Cohen, J. Feinglass, W. C. McGaghie, and D. B. Wayne, "Use of simulation-based education to reduce catheterrelated bloodstream infections," JAMA Internal Medicine, vol. 169, no. 15, pp. 1420-1423, 2009.

[19] E. R. Cohen, J. Feinglass, J. H. Barsuk et al., "Cost savings from reduced catheter-related bloodstream infection after simulation-based education for residents in a medical intensive care unit," Simulation in Healthcare, vol. 5, no. 2, pp. 98-102, 2010.

[20] Instructions for Completion of Primary Bloodstream Infection (BSI) Form (CDC 57.108), Jan 2017 [cited 2017 April 20]; 1-7, https://www.cdc.gov/nhsn/forms/instr/57_108.pdf.

[21] American Society of Anesthesiologists, "Standards \& Guidelines," 2017 [cited 2017 Sep. 19]; 1-9, http://www.asahq.org/quality-and-practice-management/standards-and-guide-lines/search?q=ASA $\% 20$ PS.

[22] A. Y. Noaman, F. Nadeem, A. H. M. Ragab et al., "Improving prediction accuracy of 'central line-associated blood stream infections' using data mining models," BioMed Research International, vol. 2017, Article ID 3292849, 12 pages, 2017. 


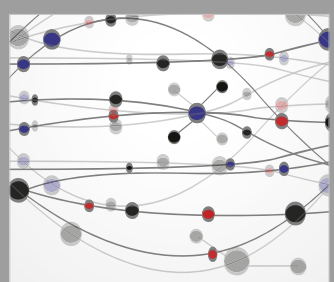

The Scientific World Journal
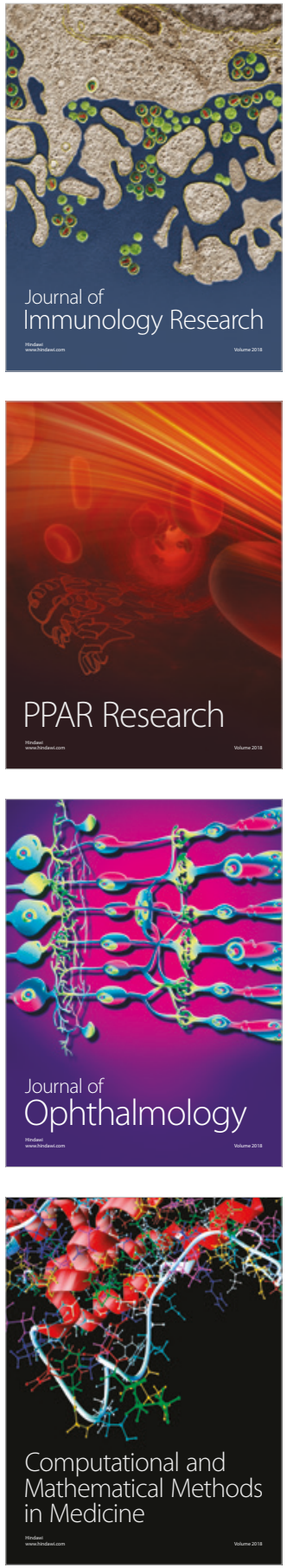

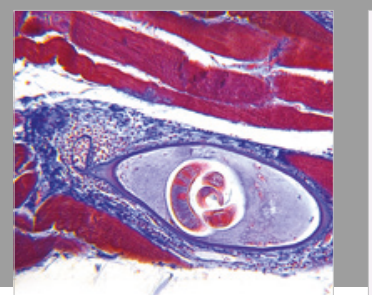

Gastroenterology Research and Practice

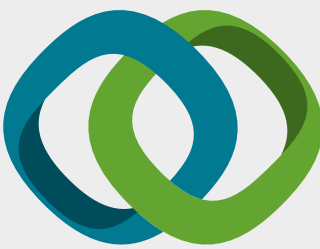

\section{Hindawi}

Submit your manuscripts at

www.hindawi.com
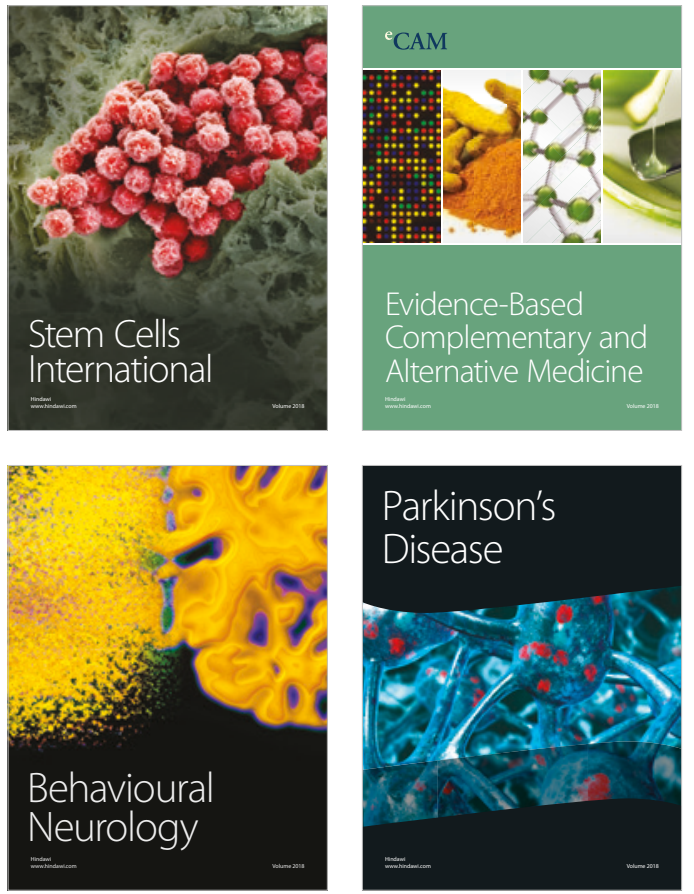

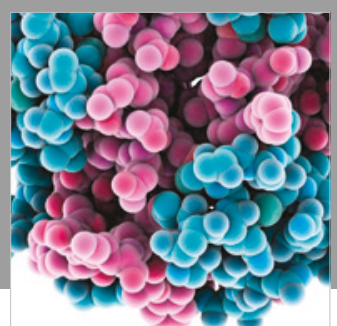

ournal of

Diabetes Research

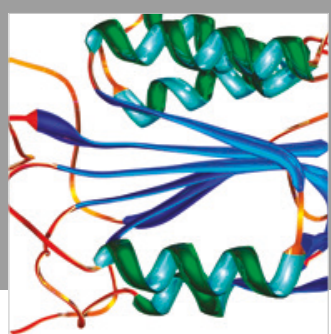

Disease Markers
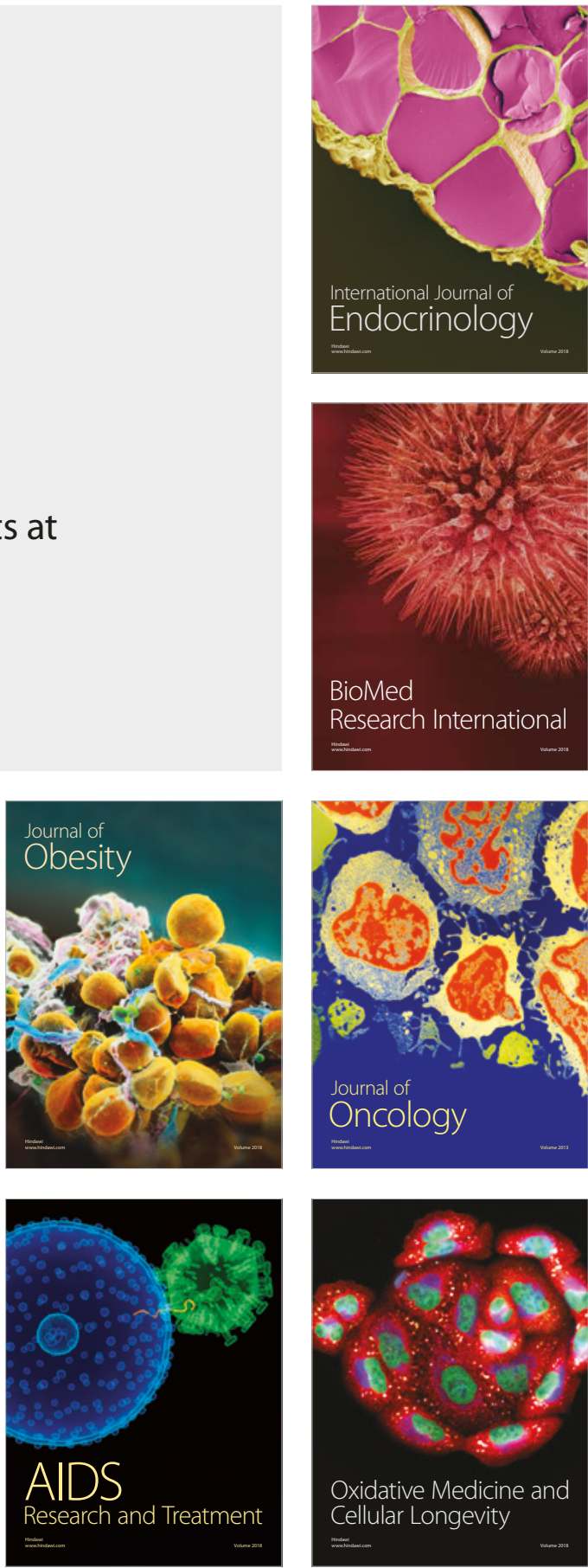